Blancquaert, I. 27

Brand, A. 5, 67

Brand, H. 67

Croucher, P.J.P. 55

Dabrock, P. 34

El Mokhtari, N.E. 55

Epplen, J.T. 62

Feuerstein, G. 50

Gwinn, M. 21

Holtzman, N.A. 8

Khoury, M.J. 21
Kollek, R. 50

Krawczak, M. 55

Kunstmann, E. 62

Nikolaus, S. 55

Schmedders, M. 50

Schreiber, S. 55

Schröder, P. 5, 67

van Aken, J. 50

van den Daele, W. 40

von Eberstein, H. 55

Zimmern, R. 67

\title{
Subject Index Vol. 9, No. 1, 2006
}

Adverse drug reactions 50

Autonomy 34

Biobank 55

Biobanks 21

Capabilities approach 34

Collaborative research 27

Common welfare 34

Complex diseases 55

Decision-making 27, 62

Diagnostic tools 62

Directive counseling 40

Ethical aspects 34

European network 67

Genetic epidemiology 55

- testing 62

Genetics 8, 27

Genomics 21

- and population health 67

Genotype-phenotype relationship 55

Health technology assessment 27
International collaborations 67

Knowledge transfer 27

Mandatory genetic testing 40

Newborn screening 8

Partnerships 27

Pharmacogenetics 50

Policy making 67

Population-based sampling 55

- studies 21

Pre-employment screening 40

Public health 8, 21, 50, 62

- health genetics 67

Relative risk 55

Resource allocation 8

Selective abortion 40

Social justice 34

Testing 50

Translation 21

Voluntary compliance 40 